\title{
Studying suicide from the life course perspective: implications for prevention
}

\author{
D. GUNNELL and G. LEWIS
}

\begin{abstract}
Summary Suicide is an important contributor to premature mortality accounting for over 800000 deaths worldwide every year. Environmental and genetic factors acting from before birth to old age affect an individual's risk of suicide. Risk is influenced not only by psychiatric illness and impulsive behaviour but also by factors such as the cultural acceptability of suicide, the ease of availability of lethal suicide methods, help-seeking behaviours in times of crisis and access to effective treatments following self-harm. Suicide prevention programmes might usefully focus on two discrete areas: the prevention of the psychiatric illnesses that precede suicide and tackling those risk factors particular to suicide such as media influences, help-seeking, the availability of methods and the medical management of self-harm.
\end{abstract}

\section{Delcaration of interest None.}

Suicide is an important contributor to global patterns of mortality, accounting for over 800000 deaths a year (World Health Organization, 2002). Since the publication of the government's suicide prevention strategy (Department of Health, 2002), it has become a key focus for psychiatric services in the UK.

Unlike many causes of death, suicide is not the result of a single disease process. It occurs as a consequence not only of a range of psychiatric disorders - most commonly depression, substance misuse and schizophrenia - but also of impulsive behaviour in moments of crisis and in the context of serious physical illness (Fig. 1). In this editorial we consider whether the study of suicide contributes important aetiological and preventive insights over and above those gained by studying its 'constituent' disorders (i.e. depression, schizophrenia, etc). We then summarise research evidence for the influence on suicide risk of factors acting at different stages of the life course; this evidence is part of a growing body of literature suggesting that prenatal and childhood exposures, as well as adult risk factors, may modify an individual's health in adulthood (Kuh \& Ben Shlomo, 1997). These influences are integrated into a model of suicidal behaviour that may facilitate a more focused approach for suicide prevention strategies.

\section{WHY STUDY SUICIDE RATHER THAN ITS 'COMPONENT' DISORDERS?}

Although suicide is unusual in the absence of mental distress, most people with psychiatric disorder and/or suicidal thoughts do not kill themselves. Other triggers or vulnerability factors contribute to risk. For example, in the Christchurch cohort, a discrete set of risk factors - including sexual abuse, a family history of self-harm and low educational achievement appeared to influence vulnerability to suicidal behaviour among individuals with depression (Fergusson et al, 2003).

Several other observations indicate that suicide results from factors over and above those causing psychiatric disorder. First, the incidence of depression - the most common antecedent of suicide - is higher in women than in men, whereas in most countries suicide occurs 3-4 times more frequently in men. Several factors may account for these differences, including stronger social bonds (particularly motherhood) in women, gender differences in help-seeking behaviour and gender differences in preferred methods of suicide - women tend to favour less lethal methods such as self-poisoning. Second, there are some discrete neurobiological features of suicidal behaviour (such as deficiency of serotonergic function) that appear to be associated with suicide, regardless of the underlying psychiatric disorder (Mann, 1998). Third, there are ten-fold differences in national suicide rates within Europe that are unlikely to be due to differences in the prevalence of mental disorder. Variations in the cultural acceptability of suicide, socioeconomic conditions, legal definitions of suicide and, possibly, genetic differences are likely to contribute to international variations. Fourth, an important influence on an individual successfully acting on a suicidal impulse is the availability of lethal methods of suicide (Clarke \& Lester, 1989); this observation is borne out by the fluctuating levels of suicide in Samoa in the 1970s and 1980s, which closely mirrored changes in imports of paraquat, a highly toxic pesticide commonly used as a method of suicide in developing nations (Bowles, 1995). Finally, case fatality following a suicide attempt is influenced not only by choice of method but also by access to effective treatments. For example, reductions in mortality from paracetamol overdose followed the introduction of $\mathrm{N}$-acetylcysteine in the UK (Flanagan \& Rooney, 2002), and in Sri Lanka monthly fluctuations in case fatality rates for selfpoisoning mirrored the availability of appropriate antidotes (Eddleston et al, 2003). It is noteworthy that most suicide prevention strategies pay little attention to the medical management of self-harm. This neglect is understandable for methods of suicide that result in death soon after the attempt is made, such as hanging and use of firearms, but is less so for other methods of suicide, particularly self-poisoning, where appropriate medical treatment may be life-saving.

Suicide prevention therefore depends not only on an understanding of how to prevent psychiatric disorder, but also on knowledge concerning how other social, economic and medical factors affect suicide risk.

\section{PRE-ADULT AND GENETIC INFLUENCES ON SUICIDE RISK}

A few studies have examined associations of suicide with indirect markers of preadult adversity - birth weight and height. Influences on body growth may also 


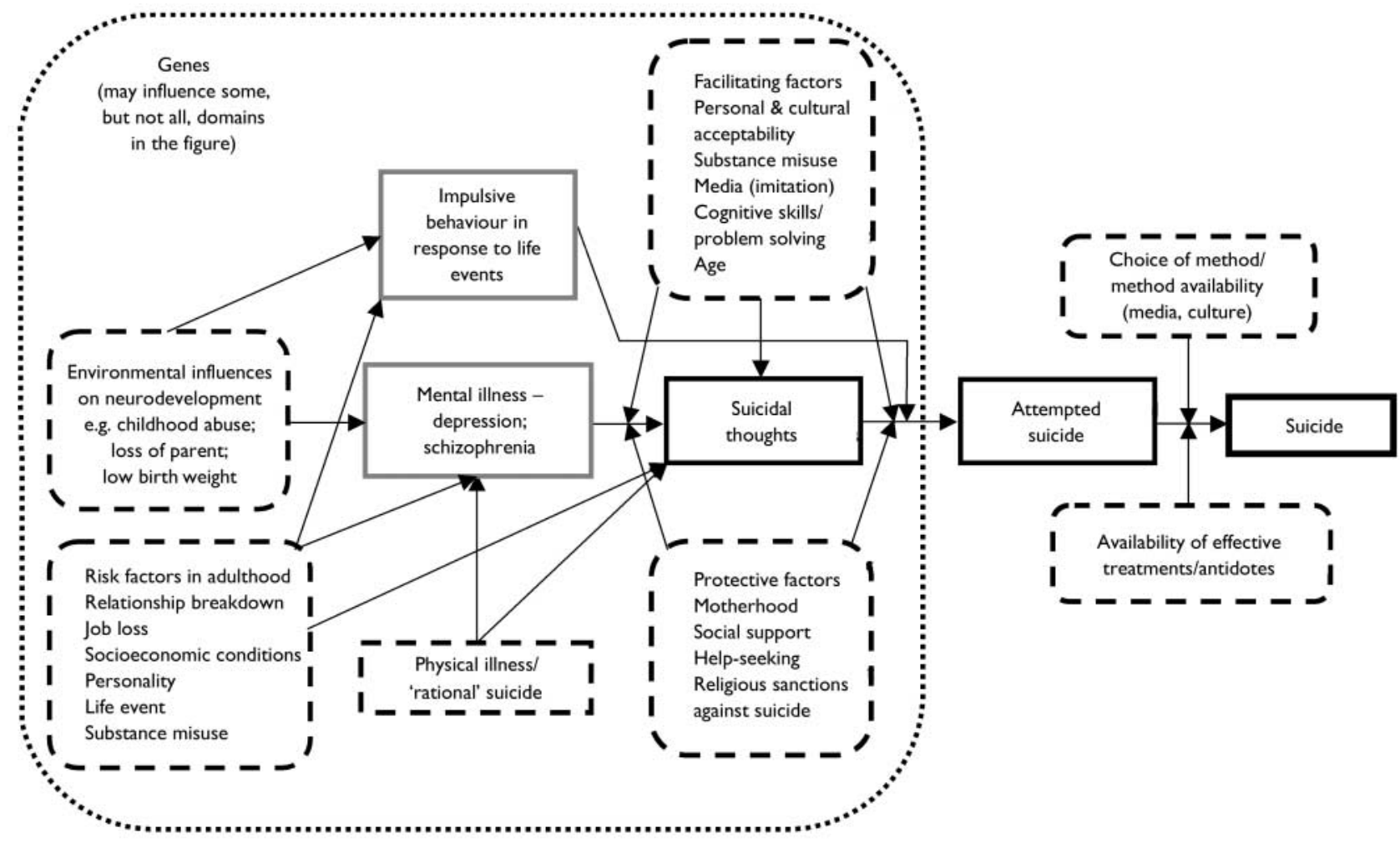

Fig. I Life course influences on suicide.

affect neurodevelopment and the risk of developing mental illness. Birth weight is a marker of foetal growth and may be influenced by maternal nutrition, body size, smoking, health and socio-economic position. Height, as well as being influenced by genes, is influenced by diet, health and psychosocial adversity throughout the growing years (Mascie-Taylor, 1991). An emerging literature suggests that there are associations of suicide with low birth weight (Mittendorfer-Rutz et al, 2004), poor infant growth (Barker et al, 1995) and short stature (Magnusson et al, 2005). Explanations for these associations are unclear. They may not be independent of the associations of these measures with depression, schizophrenia and other psychiatric disorders (Thompson et al, 2001; Cannon et al, 2002). Programming of the hypothalamic-pituitary axis has been suggested as a possible explanation for observed associations of suicide with measures of growth in early life (Barker et al, 1995).

As already described, vulnerability to self-harm may be increased by factors such as a history of childhood sexual abuse and a family history of self-harm (Fergusson et al,
2003). The association with a family history of self-harm highlights the importance either of genetic susceptibility or of behaviours learnt from other family members - or a combination of the two phenomena. Twin and adoption studies indicate that there is a genetic component to suicide. Although a number of brain pathways have been investigated, the strongest evidence is for a role of the serotonergic system (Mann, 1998). This pathway is also implicated in aggressive and impulsive behaviours, both of which may contribute to suicidal behaviour. It is likely, although not proved, that the effects of environmental stressors on suicide risk may differ in those with and those without genetic predisposition to suicidal behaviour.

Psychological mechanisms underlying associations of childhood adversity with suicidal behaviour are not fully understood. A possible pathway is through the effects of childhood trauma on the memory system. The hypothesis has been put forward that traumatic events in childhood result in impaired autobiographical memory, which impairs problem-solving and may result in suicidal behaviour in times of crisis (Williams \& Pollock 2000).

\section{ADULT INFLUENCES ON SUICIDE}

The main psychiatric disorders contributing to suicide risk in adults are depression and schizophrenia. A number of social and economic factors - unemployment, divorce, serious medical illness and substance misuse - are also commonly associated with suicide. In contrast, parenthood and being in a stable relationship appear to be protective (Qin et al, 2003).

One problem that besets observational research into the relative importance of mental illness and socio-economic risk factors for suicide is our limited understanding of the nature of the underlying causal pathways for these associations. For example, Danish studies suggest that there is only a weak association between unemployment and suicide after controlling for an individual's past psychiatric history among other exposures (Mortensen et al, 2000). This may be interpreted as suggesting that people with psychiatric illness are 
more likely to lose their job or find it difficult to gain employment, and that this explains the association of unemployment with suicide. However, analysis of temporal trends in unemployment and suicide shows that rises in unemployment are associated with increases in suicide (Gunnell et $a l, 1999)$. It seems improbable that the rises in unemployment are caused by rises in mental illness. More plausibly, increases in unemployment result in greater levels of depression and distress and this in turn influences trends in suicide. Both pathways are likely to contribute to associations of suicide with unemployment. Similarly, being unmarried is associated with an increased risk of suicide (Qin et al, 2003) - but does the absence of a close relationship cause psychiatric illness, or vice versa? Multivariable analyses of large data-sets suggest that both pathways may contribute (Qin et al, 2003).

Serious physical illness in adulthood is also associated with increased suicide risk Evidence is strongest for cancer and neurological disorders such as multiple sclerosis. It is noteworthy that the increased risk of suicide associated with AIDS appears to have declined with the advent of new therapies (Stenager \& Stenager, 2000).

Religious beliefs and other cultural factors may influence the likelihood of an individual acting upon suicidal thoughts (Neeleman \& Lewis, 1999). The reporting and portrayal of fictional or actual suicides in the media has also been found to influence patterns of suicidal behaviour (Hawton \& Williams, 2002). The media and religious beliefs may influence both the acceptability of suicide and an individual's choice of method of suicide. More crudely, changing availability of particularly lethal methods of suicide may influence rates. In Britain, the decreased lethality of domestic gas, the most commonly used method of suicide in the $1960 \mathrm{~s}$, is thought to have contributed to the decline in the national suicide rate at this time (Kreitman, 1976).

\section{CONCLUSIONS}

Genetic and environmental factors acting at different stages of the life course are associated with the risk of suicide. Such risk factors are more extensive than those of relevance to the aetiology of psychiatric disorder. An individual's risk is also influenced by the chosen suicide method and the

D. GUNNELL, Department of Social Medicine, G. LEWIS, Division of Psychiatry, University of Bristol, Bristol, UK

Correspondence: David Gunnell, Department of Social Medicine, University of Bristol, Canynge Hall, Whiteladies Road, Bristol BS8 2PR, UK. Tel: +44 (0) 117928 7235; e-mail D.J.Gunnell@bristol.ac.uk

(First received 9 December 2004, final revision 17 February 2005, accepted 24 February 2005)

availability of medical services to manage the consequences of the suicide attempt. Figure 1 illustrates the possible pathways through which factors acting from before birth to immediately proximal to the suicide attempt might influence an individual's decision to attempt suicide and the outcome of such a decision. A fuller understanding of factors operating across the life course that either protect against or precipitate suicide, as well as the key periods of development when they act, will provide useful insights into its prevention.

The evidence reviewed here suggests that national suicide prevention programmes might usefully define two discrete areas of work. The first should focus on the prevention of psychiatric disorder. The second should focus on risk factors particular to suicide, such as media influences and the availability of methods of suicide and medical treatment. Striking a balance between these two approaches is important and there might be times when the two would conflict. For example, although prevention of suicide is a priority for all mental health services, there is a possibility that some methods used to reduce risk in those with psychiatric disorder, such as increased in-patient observation and removal of belts and shoelaces, could reduce the therapeutic value of in-patient treatment. In the wider community, prevention of suicide by reducing access to means alone does not address the root cause of distress that results in some people taking their own lives, and does not necessarily signal an improvement in the mental health of the wider population.

\section{REFERENCES}

Barker, D. J., Osmond, C., Rodin, I., et al (1995) Low weight gain in infancy and suicide in adult life. BMJ, 3II, 1203

Bowles, J. R. (1995) Suicide in Western Samoa: an example of a suicide prevention program in a developing country. In Preventative Strategies on Suicide (eds R. F.W. Diekstra, et al), pp. 173-206. Leiden: Brill.

Cannon, M., Jones, P. B. \& Murray, R. M. (2002) Obstetric complications and schizophrenia: historical and meta-analytic review. American Journal of Psychiatry, 159, 1080-1092

Clarke, R.V. \& Lester, D. (1989) Suicide: Closing the Exits. New York: Springer.
Department of Health (2002) National Suicide Prevention Strategy for England. London: Department of Health.

Eddleston, M., Senarathna, L., Mohamed, F., et al (2003) Deaths due to absence of an affordable antitoxin for plant poisoning. Lancet, 362, I041-1044.

Fergusson, D. M., Beautrais, A. \& Horwood, L. J. (2003) Vulerability and resiliency to suicidal behaviours in young people. Psychological Medicine, 33, 61-73.

Flanagan, R. J. \& Rooney, C. (2002) Recording acute poisoning deaths. Forensic Science International, 128, 3-19.

Gunnell, D., Lopatatzidis, A., Dorling, D., et al (1999) Suicide and unemployment in young people. Analysis of trends in England and Wales, 1921-1995. British Journal of Psychiatry, 175, 263-270.

Hawton, K. \& Williams, K. (2002) Influences of the media on suicide. BMJ, 325, 1374-1375.

Kreitman, N. (1976) The coal gas story. British Journal of Preventive and Social Medicine, 30, 86-93.

Kuh, D. \& Ben Shlomo, Y. (1997) A Lifecourse Approach to Chronic Disease Epidemiology. Oxford: Oxford University Press.

Magnusson, P. K. E., Gunnell, S., Tynelius, P., et al (2005) Strong inverse association between height and suicide in a large cohort of Swedish males. Evidence of early life origins of suicidal behaviour? American Journal of Psychiatry, 162, 1373-1375.

Mann, J. J. (1998) The neurobiology of suicide. Nature Medicine, 4, 25-30

Mascie-Taylor, C. G. N. (199I) Biosocial influences on stature: a review. Journal of Biosocial Science, 23, 113-128.

Mittendorfer-Rutz, E., Rasmussen, F. \& Wasserman, D. (2004) Restricted fetal growth and adverse maternal psychosocial and socioeconomic conditions as risk factors for suicidal behaviour of offspring: a cohort study. Lancet, 364, II35-1140.

Mortensen, P. B., Agerbo, E., Erikson, T., et al (2000) Psychiatric illness and risk factors for suicide in Denmark. Lancet, 355, 9-12.

Neeleman, J. \& Lewis, G. (1999) Suicide, religion, and socioeconomic conditions. An ecological study in 26 countries, 1990. Journal of Epidemiology and Community Health, 53, 204-210.

Qin, P., Agerbo, E. \& Mortensen, P. B. (2003) Suicide risk in relation to socioeconomic, demographic, psychiatric, and familial factors: a national register-based study of all suicides in Denmark, 1981-1997. American Journal of Psychiatry, 160, 765-772.

Stenager, E. N. \& Stenager, E. (2000) Physical illness and suicidal behaviour. In International Handbook of Suicide and Attempted Suicide (eds K. Hawton \& K. van Heeringen), pp. 405-420. Chichester: Wiley.

Thompson, C., Syddall, H., Rodin, I., et al (200I) Birth weight and the risk of depressive disorder in late life. British Journal of Psychiatry, 179, 450-455

Williams, J. M. G. \& Pollock, L. R. (2000) The psychology of suicidal behaviour. In International Handbook of Suicide and Attempted Suicide (eds K. Hawton \& K. van Heeringen), pp. 79-93. Chichester: John Wiley.

World Health Organization (2002) The World Health Report 2002. Reducing Risks, Promoting Healthy Life. Geneva: WHO 